\title{
Energy Transfer Processes in Solar Energy Conversion (Grant \#DE-FG03-84ER13251)
}

\author{
Michael D. Fayer \\ Department of Chemistry \\ Stanford University, Stanford, CA 94305-5080
}

November 1989

(Progress Report covers the current three year funding period.)

\section{DISCLAIMER}

This report was prepared as an account of work sponsored by an agency of the United States Government. Neither the United States Government nor any agency thereof, nor any of their bility for the accurany warranty, express or implied, or assumes any legal liability or responsibility for the accuracy, completeness, or usefulness of any information, apparatus, product, or ence herein to any, or represents that its use would not infringe privately owned rights. Reference herein to any specific commercial product, process, or service by trade name, trademark, manufacturer, or otherwise does not necessarily constitute or imply its endorsement, recommendation, or favoring by the United States Government or any agency thereof. The views United States Government or any agency thereof. necessarily state or reflect those of the 
During the past three years we have made substantial progress in experimental and theoretical studies in two areas: Photoinduced donor to acceptor electron transfer followed by back transfer in random solutions; and electronic excitation transport in systems with complex inhomogeneous spatial geometries and inhomogeneous energy distributions. Through the development of accurate statistical mechanical theories, we have been able to relate dynamics in complex systems to experimental observables. We have then used the experimental observables, time resolved fluorescence depolarization and transient grating experiments, to examine well defined molecular systems. As is discussed below, the agreement between theory and experiment is excellent. The work during the current funding period sets the stage for a new round of advances which are discussed in the accompanying proposal.

In addition to the brief progress report, a list of publications supported by this grant is provided. Considerable detail is also given in the proposal, so it should be unnecessary to examine the published papers to obtain a clear understanding of the past and future work.

\section{Photoinduced Donor-Acceptor Electron Transfer and Back Transfer in Solution}

\section{A. Solid Solutions}

A photoexcited donor can transfer an electron to an acceptor. In a random solution, each donor is surrounded by many acceptors. Once the electron has transferred, there exists a cation in close proximity to an anion. Since the thermodynamically lowest energy state is the ground state neutrals, the electron will back transfer from the anion to the cation. In liquid solutions, there is the possibility that the ions will separate by diffusion prior to back transfer. The radical ions are very reactive and 
meaningful molecular parameters from the experimental results.

While the forward transfer process is relatively straightforward to study using time resolved fluorescence quenching, the back transfer process requires the application of a method which is sensitive to the ground state ion concentrations. We demonstrated that a picosecond transient grating (TG) experiment is ideally suited for observation of back transfer dynamics. The grating excitation pulses excite the donor molecules. This produces an excited state grating. Electron transfer turns the excited state grating into a grating of ion pairs. Back transfer quenches the grating. The time dependent TG signal is determined by the forward transfer parameters and the back transfer parameters, as well as the acceptor concentration and the donor excited state life time. The forward transfer parameters are obtained from time resolved and steady state fluorescence measurements. These are used with the theory and the TG experimental results to yield the back transfer parameters.

In the experiments, we studied rubrene (donor) and duroquinone (acceptor) in sucrose octaacetate glass. A wide variety of acceptor concentrations were studied and great care was taken to avoid all possible experimental artifacts. Excellent agreement between theory and experiment was obtained. In fact, the theoretical and experimental curves were essentially indistinguishable. Using the measured parameters and the theoretical expressions for the ion probabilities, it is possible to calculate a number of interesting time dependent properties that are characteristic of electron transfer and back transfer in an ensemble of donors and acceptors randomly distributed in a rigid solution. Calculations of the time dependent ion populations and the probability that the $i^{\text {th }}$ acceptor is an anion as a function of time and distance were 
Back transfer at short time can be substantially faster than back transfer once solvent relaxation is complete. Therefore it is important to include solvent relaxation effects in back transfer, and a study of the time dependent rate of back transfer can be used as a probe of solvent relaxation.

Solvent relaxation times are typically much shorter than 100 ps. Thus on the time scale of the experiments mentioned above the solvent relaxation was complete, and a time independent electron transfer rate was observed. However, at times shorter than 100 ps, a femtosecond resolution transient grating experiment should be sensitive to changes in the observables due to solvent relaxation.

We have included solvent relaxation in the theory previously developed for forward and back electron transfer. We use a simple, yet reasonable, model for solvent relaxation, but any model for the solvent dynamics that gives an electron transfer rate which evolves in time can be used. The essence of the problem is to include the time dependent back transfer rate into the averages over the distance dependence of the forward and back transfer rates.

A distribution function for the time dependent ion probability in various solvent states was derived. Next a time and distance dependent back electron transfer rate was obtained. The rate has a distance dependent part that falls off exponentially with distance and a part that depends on the amount of solvation, which is similar to the Marcus' theory exponentially activated rate. Finally, the total ion probability funetion was derived and the ensemble averaged.

Using these results, the ion probability as a function of time and distance was calculated for a variety of solvent relaxation times and 
solution (molecular positions fixed), we were able to obtain an exact solution to the time dependent problem by converting the many particle problem into a two particle problem using an interesting approach to doing the statistical mechanical averages. This method provides a mathematically tractable starting point for solving the problem with diffusion of the particles included.

The forward electron transfer problem with diffusion has been treated in the literature in two limiting cases, very fast diffusion and very slow diffusion. To treat the forward and back transfer problem for any rate of diffusion (any diffusion constant D), it was first necessary for us to extend the work on the forward transfer problem. We have been able to obtain results for any D. Using these results and extending our solid solution theoretical method to include diffusion we obtained a complete description of forward and back transfer dynamics with any $D$. A more quantitative discussion of these results is given in the accompanying proposal.)

The theory allows us to calculate the time dependent ion probabilities and properties such as the time dependence of finding ions with particular separations as a function of the forward and back transfer parameters and the diffusion constant, D. Diffusion has a substantial influence on the results.

One of the most important properties to consider is the escape probability, i.e., the probability that a cation and anion pair separate by diffusion prior to electron recombination. Only those ion pairs which separate can go on to do useful chemistry. From a formal, statistical mechanical view, none of the ions escape recombination because in an 
transport among chromophores attached to polymer chains.

The qualitative relationship between excitation transport dynamics and the volume occupied by a polymer chain (characterized, for example, by the root-mean-squared ensemble averaged radius of gyration, $\left\langle R_{g}{ }^{2}\right\rangle^{1 / 2}$ ) is straightforward. Consider an ensemble of polymer chains, with chromophores bound to the chains, dispersed in a host matrix at low enough concentration that the chromophore tagged chains are isolated from each other. These chains will have an $\left\langle\mathrm{R}_{\mathrm{g}}{ }^{2}\right\rangle^{1 / 2}$ determined by the guest-host interactions. If the guest chains are dispersed in a host polymer with which they have favorable thermodynamic interactions, they will assume extended configurations with a large average interchromophore separation. Since the rate of excitation transport is a strong function of distance (proportional to $1 / r^{6}$ where $r$ is the chromophore separation), an extended conformation results in a relatively slow rate of excitation transport. Conversely, if the same guest polymer is placed in a different host, where thermodynamic interactions are less favorable, the chains will contract, resulting in more rapid excitation transport. The $1 / r^{6}$ distance dependence makes excitation transport observables very sensitive to small changes in $\left\langle\mathrm{R}_{\mathrm{g}}{ }^{2}\right\rangle^{1 / 2}$. Clearly, an experiment which monitors the rate of excitation transport among chromophores on polymer chains will be directly related to the chain size and polymer-polymer interactions.

We have been able to quantitatively examine excitation transport in polymers and other complex systems by applying a statistical mechanical theory for excitation transport which is mathematically tractable enough to permit analysis of complex spatial chromophore geometries. The theory is based on a first order cummulant expansion solution to the transport master 
In addition, concentration studies show that the technique is extremely sensitive for detecting micro-phase separation on the order of the aggregation of two or three chains. When the concentration of chromophores containing chains is increased in an incompatible blend (poor solvent), aggregation can occur. This results in microdomains which contain many chains with chromophores. The aggregates have very high local chromophore concentrations which leads to very fast excitation transport. Because the chromophores are bound to the polymer backbones, the formation of dimers and $n$-mers, which quench excited states, is prevented. Further details are given in the proposal.

In addition to studying chains which are tagged with chromophores along the backbone, we have developed a detailed statistical mechanical model for energy transport on short polymer chains which are end tagged with chromophores. Short chains do not obey Gaussian statistics. Also to properly describe end tagged chains it is necessary to include the nature of the chemical linkage which connects the chromophore to the chain ends. We accomplished this by developing distribution functions for the end to end distances of random walks with a small number of steps (2-20) with variable step size. This was then used in the excitation transport calculations.

B. Dispersive Excitation Transport At and Near Room Temperature At or near room temperature the excitation transfer rate has been assumed to be independent of chromophore energy. For singlet excited states the distance dependence of the transfer rate is governed by a dipole-dipole interaction. The quantum mechanics of the energy transfer step is incorporated into a single pairwise, distance dependent transfer 
electronic transition energies an excitation not only moves spatially among the chromophores, but it moves to lower energy as it is transferred. The excitation will tend to move to the red edge of the absorption line by a series of transfer steps to lower energy chromophores while emitting phonons (heat) to conserve energy.

Recently, we reported the first observation of dispersive electronic excitation transport at and near room temperature by monitoring the timeresolved fluorescence depolarization from an ensemble of chromophores. The systems studied were random copolymers of 2 -vinylnaphthalene and methyl methacrylate dissolved in small concentration in bulk poly(methyl methacrylate). Measurements were made at a number of excitation wavelengti. and sample temperatures. The sensitivity of this experimental technique to the details of the time dependence of excitation transfer made it possible to reveal the energy dependent transfer among chromophores for which the homogeneous and inhomogeneous linewidths are similar. A theory was developed to model the experimental results in terms of known quantities about this system. It was found that the experimental findings could be explained essentially quantitatively by modifying the theory of Peterson and Fayer for non-dispersive excitation transfer among chromophores randomly attached to a polymer chain, with details of the spectral characteristics of the chromophores and assumptions about the mechanism of dispersive energy transfer.

The experiments were extended to include measurements of dispersive transport in a simpler system, 2-ethylnaphthalene (2-EN) molecules randomly distributed in PMMA. It was found that the free naphthyl chromophores have a different inhomogeneous linewidth than when attached to a polymer chain. The 2 -EN in PMMA experiments show that dispersive energy transport at high 
1. "Experimental Investigation on Donor-Acceptor Electron Transfer and Back Transfer in Solid Solutions," R. C. Dorfman, Y. Lin, and M. D. Fayer, J. Phys. Chem., 93, 6388 (1989).

2. "Time Dependence of Donor-Acceptor Electron Transfer and Back Transfer in Solid Solution," Y. Lin, R. C. Dorfman, and M. D. Fayer, J. Chem. Phys., 90, 159 (1989).

3. "Photoinduced Electron Transfer and Back Transfer in Systems of Randomly Distributed Donors and Acceptors: Picosecond Transient Grating Experiments," R. C. Dorfman, Y. Lin, M. B. Zimmt, J. Baumann, R. P. Domingue, and M. D. Fayer, J. Phys. Chem., 92, 4258 (1988).

4. "Solvent Relaxation and Electron Back Transfer Following Photoinduced Electron Transfer in an Ensemble of Randomly Distributed Donors and Acceptors," Y. Lin, R. C. Dorfman, and M. D. Fayer, J. Chem. Phys. submitted (1989).

5. "Dispersive Excitation Transport at Elevated Temperatures ( $50 \mathrm{~K}$ $298 \mathrm{~K})$ : Experiments and Theory," Alan D. Stein, Kristen A. Petersen, and M. D. Fayer, J. Chem. Phys. submitted (1989).

6. "Dispersive Electronic Excitation Transport in Polymeric Solids at and near Room Temperature," Alan D. Stein, Kristen A. Peterson, and M. D. Fayer, Chem. Phys. Lett., 161, 16 (1989).

7. "Quantitative Measurement of Chain Contraction in a Solid Blend of Two Incompatible Polymers: Poly(Methyl Methacryalte)/Poly(Vinyl Acetate)," Kristen A. Petersen, Alan D. Stein, and M. D. Fayer, Macromolecules, accepted (1989).

8. "Quantitative Determination of the Radius of Gyration of Poiy(Methyl Methacrylate) in the Amorphous Solid State by Time-Resolved Fluorescence Depolarization Measurements of Excitation Transport," K. A. Peterson, M. B. Zimmt, S. Linse, R. P. Domingue, and M. D. Fayer, Macromolecules 20, 168 (1987).

9. "Short Polymer Chain Statistics and the Relationship to End to End Electronic Excitation Transport: Random Walks with Variable Step Lengths," M. B. Zimmt, K. A. Peterson, and M. D. Fayer, Macromolecules, 21, 1145 (1988).

10. "Fluorescence Depolarization of Chromophores in Polymeric Solids," K. A. Peterson, M. B. Zimmt, M. D. Fayer, Y. H. Jeng, and C. W. Frank, Macromolecules, 22, 874 (1988).

11. "Electronic Excitation Transport in Restricted Geometries," Kristen A. Peterson, Alan Stein, and M. D. Fayer in Molecular Dynamics in Restricted Geometries, Eds. J. Klafter and J. M. Drake (John Wiley \& Sons, New York). 

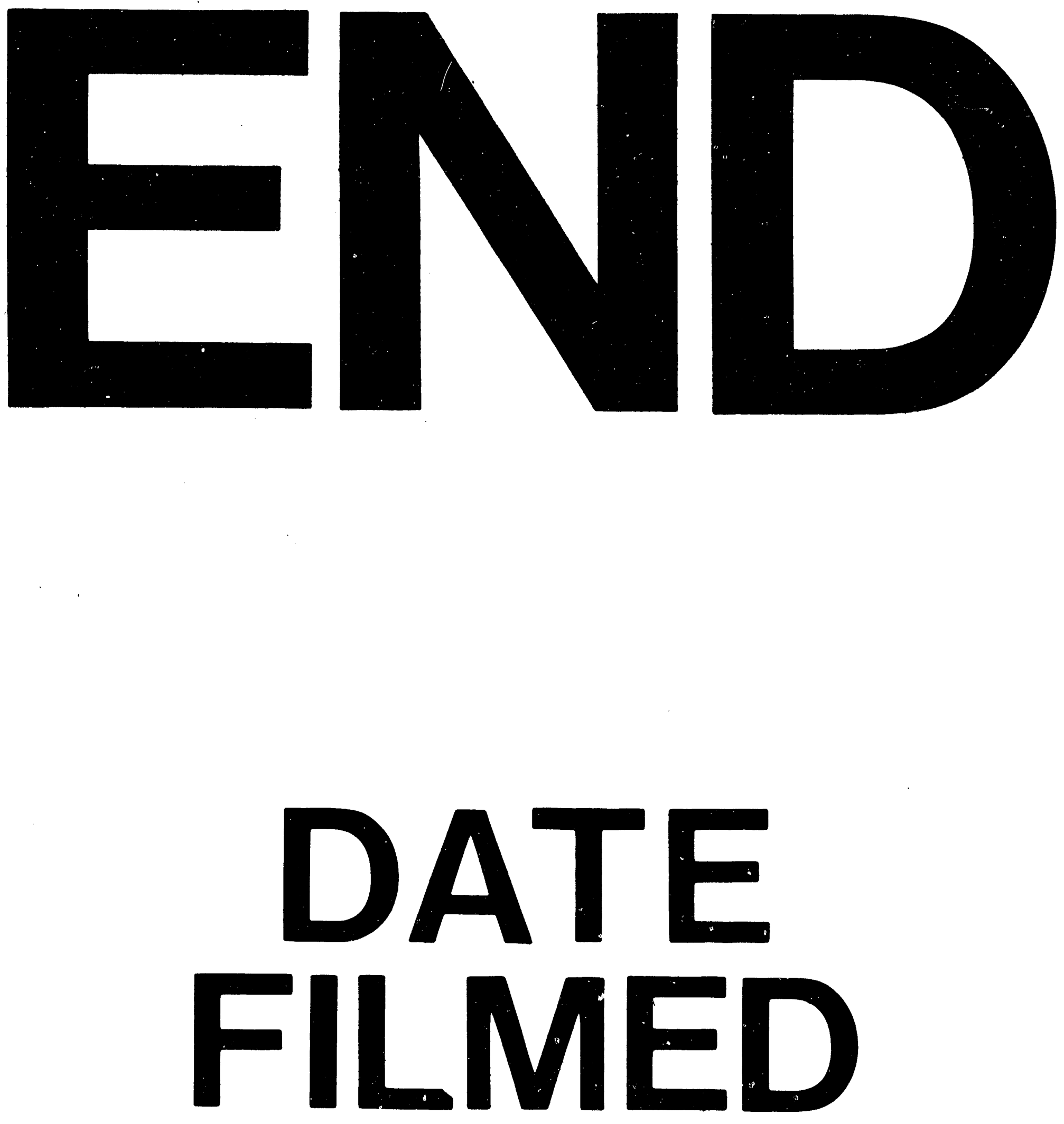

f

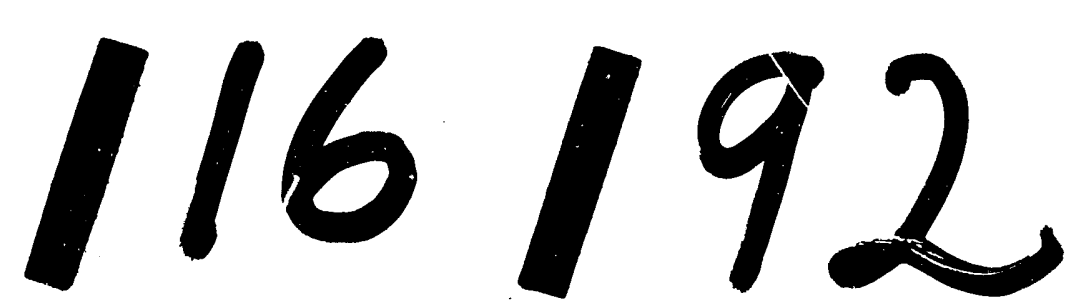

$=$ 
$\mid$ 